\title{
Estudio descriptivo de los pacientes ambulatorios con enfermedad hepática por alcohol en nuestro medio
}

\author{
D. LEDRO CANO, J. REBOLLO BERNÁRDEZ, Y. TORRES DOMÍNGUEZ, \\ I. CARMONA SORIA, J. GONZÁLEZ-VILCHES*, M. JIMÉNEZ SÁENZ, \\ J. M. HERRERÍAS GUTIÉRREZ
}

Unidad de Hepatología. Servicio de Aparato Digestivo. *Departamento de Bioquímica. Área Hospitalaria Virgen Macarena. Sevilla

\author{
DESCRIPTIVE STUDY OF OUTPATIENTS WITH ALCOHOL LIVER \\ DISEASE IN OUR POPULATION
}

\begin{abstract}
RESUMEN
Objetivo: Nos propusimos describir las características demográficas y los hábitos de consumo de alcohol de un grupo de pacientes ambulatorios. Intentamos discernir la influencia de la edad, sexo, habitat y nivel socioeconómico sobre el hábito enólico.

Diseño experimental: Nuestro estudio es retrospectivo, de base institucional. Pacientes: 164 pacientes ambulatorios, en seguimiento en nuestras consultas externas de la unidad de Hepatología por enfemedad hepática alcohólica.

Resultados: La edad media de inicio fue 18,6 (7,36) años; los años de enolismo medio fueron de $35,4(13,5)$ años y el consumo medio de alcohol de 161,2 (116,7) gramos de alcohol/día. Solo en 16 hombres (8\%) se observó un consumo menor de 60 gramos de alcohol al día, y 5 mujeres $(35,7 \%)$ consumían menos de 40 gramos de alcohol al día. El consumo de alcohol a lo largo de la vida estuvo correlacionado con el índice de Maddrey al final del estudio $(r=+0,407)$. De igual forma el consumo diario de gramos de alcohol estuvo correlacionado con el aspecto ecográfico del hígado( $\mathrm{r}=+0,283)$, apreciándose también correlación de dicha técnica de imagen $(r=+0,301)$ con el Tiempo de Protrombina al inicio del estudio. El porcentaje de pacientes que presentaron al menos un episodio de descompensación de su cirrosis fue del 39\%.

Conclusiones: La edad de inicio ronda la mayoría de edad . El consumo de alcohol a lo largo de la vida, estuvo correlacionado con el tiempo de protrombina en la última visita y el aspecto ecográfico del hígado.
\end{abstract}

PALABRAS CLAVE: Alcoholismo. Ambulatorios. Ecografía doppler. Índice de Maddrey. Epidemiología. España.

\section{ABSTRACT}

Aims: We tried to show the demographic characteristic and alcohol intake habits among our outpatients. We study the influence of age, sex, habitat and socioeconomical status on alcoholic habit.

Design: Retrospective and institution based study. Patients. 164 patients who were followed up for alcohol liver disease in our outpatient section.

Results: Average age to start drinking alcohol was 18.6 (7.36) years, years of alcoholism were 35.4 (13.5) years, average daily alcohol intake was 161.2 (116.7) grams of pure alcohol. Only 16 men (8\%) drank less than 60 grams a day. 5 ( $35.7 \%$ ) women drank less than 40 grams a day. Life-cumulative alcohol intake was correlated with Maddrey's score at the end of the study $(r=+0.407)$. Average daily alcohol intake was corre lated with ultrasonographic features of the liver $(r=+0.283)$, we aprecia ted that Prothrombin Time was also correlated with ultrasonographic features of the liver $(r=+0.301)$. The percentage of patients who suffer, at least one decompensation of their disease was $39 \%$.

Conclusions: Average age to start drinking is about legal age. Lifecumulative alcohol intake was related to Prothrombin Time and ultraso nographic features of the liver.

KEY WORDS: Alcoholism. Outpatient. Doppler ultrasound. Maddrey's score. Epidemiology. Spain.

Ledro Cano D, Rebollo Bernárdez J, Torres Domínguez Y, Carmona Soria I, González-Vilches J, Jiménez Sáenz M, Herrerías Gutiérrez JM. Estudio descriptivo de los pacientes ambulatorios con enfermedad hepática por alcohol en nuestro medio. An Med Interna (Madrid) 2001; 18: $569-572$

\section{INTRODUCCIÓN}

El consumo humano de alcohol parece remontarse al paleolítico, siendo utilizado desde entonces con distintos fines: religioso, lúdico, medicinal y como norma social. En 1987 España ocupaba el tercer lugar en el mundo en cuanto a consumo, estimándose que una dosis sostenida superior a los
$60 \mathrm{~g} /$ día en hombres y $40 \mathrm{~g} /$ día en mujeres puede ser un riesgo para el desarrollo de lesión hepática $(1,2)$ (esteatosis, hepatitis y cirrosis (3)), mostrando una mayor susceptibilidad las mujeres (4). La severidad de la lesión hepática se correlaciona con la cantidad total de alcohol consumido por día y con la duración del consumo excesivo, calculándose en nuestro país que más de un $7 \%$ de la población consume por encima de los

Trabajo aceptado: 02 de Julio de 2001

Correspondencia: Diego Ledro Cano. Virgen de Luján 45 4C. 41011 Sevilla. dledro@ supercable.es 
umbrales de riesgo, siendo la mortalidad global relacionada con el alcohol de un 6,1\%. En un estudio reciente, la prevalencia de esteatosis hepática por alcohol se sitúa en el 8,8\% de una población masculina, aparentemente sana, siendo un consumo superior a 40 gramos al día, una variable independiente de riesgo para el desarrollo de esteatosis hepática (5). Nuestro estudio es retrospectivo, de base institucional, y en él analizamos el consumo enólico y las características de pacientes ambulatorios, seguidos en nuestras consultas por su enfemedad hepática alcohólica.

Nos propusimos describir las características demográficas de un grupo de pacientes de nuestra área sanitaria diagnosticados de enfermedad hepática alcohólica, e intentamos discernir la influencia de la edad, sexo, habitat y nivel sociocultural sobre el hábito enólico. Analizamos la influencia de este hábito sobre: la presencia de complicaciones, los valores hematológico-bioquímicos (6) y los hallazgos ecográficos; también comparamos el estado de la función hepática mediante los valores analíticos frente a los hallazgos ecográficos.

\section{MATERIAL Y MÉTODOS}

Se recogieron 164 pacientes ambulatorios en seguimiento en nuestras consultas externas de la unidad de Hepatología por enfemedad hepática alcohólica. Los criterios de inclusión de los pacientes en el presente estudio fueron:

1. Edad superior a 18 años.

2. Consumo diario de alcohol igual o superior a $20 \mathrm{~g} / \mathrm{día}$ en mujeres (7) y $40 \mathrm{~g}$ /día en el hombre (5), y

3. Descartamos otras causas de hepatopatía crónica excepto las de causa viral. Éstos tres criterios debían cumplirse obligatoriamente. Los pacientes además debían cumplir uno o dos de los siguientes criterios: a) analítica: elevación por encima de los valores de referencia de nuestro laboratorio de VCM, GGT, y Transaminasas; y b) ecografía abdominal mostrando algún tipo de afectación hepática en forma de hiperecogenicidad, desestructuración del parénquima con bordes irregulares o nodulares. En la última revisión insistimos en investigar el hábito enólico mediante la anamnésis. Las variables demográficas evaluadas fueron: edad, sexo, habitat y nivel sociocultural según la profesión del sujeto, de tal manera que los trabajadores manuales se consideraron dentro del nivel bajo, los diplomados universitarios dentro del nivel medio y los graduados universitario de nivel superior fueron incluidos dentro del nivel alto y las correspondientes al consumo de alcohol fueron: edad de inicio del hábito enólico, consumo diario de gramos de alcohol (ml alcohol x 0,8 x grados de alcohol /100), años de enolismo y años de abstinencia. También investigamos el motivo de consulta, la presencia de patología psiquiátrica asociada, y la presencia de complicaciones relacionadas o no con su hepatopatía. En la última visita, valoramos en la ecografía abdominal con doppler: el aspecto del hígado (normal, esteatosis, hepatopatía crónica y cirrosis, acorde con el informe del ecografista, sin la confirmación histológica; para algunos análisis estadísticos, esta variable cualitativa se transformó en cuantitativa ordinal: $0,1,2$ y 3 , en orden ascendente según lo evolucionado de la enfermedad), el calibre y la velocidad de la vena porta, la presencia de litiasis y el tamaño del bazo. También se analizaron parámetros de laborato- rio de función hepática e indirectos del consumo de alcohol restrospectivamente evaluamos la ecografía abdominal de la primera visita así como los valores de laboratorio correspondientes a esa visita.

\section{ESTADÍSTICA}

Comenzamos con un estudio descriptivo. Para analizar la asociación entre variables cuantitativas utilizamos las correlaciones, y para analizar la asociaciones entre variables cuantitativas y cualitativas, la t de Student. El nivel de significación estadística exigido fue $\mathrm{p}<0,05$.

\section{RESULTADOS}

La edad media de los pacientes es de 55,6 (11,5) años, existiendo un mayor porcentaje de hombres con un $91.5 \%$ (150 pacientes) quizás debido a que la mujer oculta el hábito enólico. La media de años de seguimiento en las consultas externas fue 3,1 $(2,6)$ años y los principales motivos de consulta fueron: ascitis en 40 sujetos $(24,3 \%)$, signos de hepatopatía crónica en 32 sujetos $(19,5 \%)$, hipertransaminasemia en 27 sujetos $(16,4 \%)$, hemorragia digestiva alta en 16 sujetos $(9,7 \%)$, ictericia en $8(4,8 \%)$, plaquetopenia en $8(4,8 \%)$, coagulopatía en $8(4,8 \%)$, pancreatitis aguda en 5 pacientes $(3 \%)$, hepatitis aguda en $5(3 \%)$, encefalopatía hepática en $3(1,8 \%)$, elevación de la GGT en $3(1,8 \%)$, hiponatremia en $3(1,8 \%)$, existencia de LOE hepática en $3(1,8 \%)$ y anemia en $3(1,8 \%)$. Hemos observado que la edad media de inicio del hábito enólico es de 18,6 (7,36) años, siendo los años de enolismo medio de $35,4(13,5)$ años y el consumo medio de alcohol de 161,2 (116,7) gramos de alcohol/día, si bien 12 hombres $(8 \%)$ consumían menos de $40 \mathrm{~g} /$ día de alcohol y ninguna mujer consumió menos de 20 g/día de alcohol. Se aprecia una ligera prevalencia de pacientes que viven en el medio rural (93 $(56,7 \%))$ con respecto al urbano $(71(43,3 \%))$ sin significación estadística. Del total de pacientes, 120 tenían un nivel sociocultural bajo $(73,1 \%)$ y 44 medio $(26,9 \%)$. El número de pacientes que presentaban AgHBs positivo era $15(9,1 \%)$, a nueve de ellos, le fue solicitado el DNA viral, siendo positivo en 7 casos $(77,7 \%)$. En seis de estos pacientes, no fue solicitado el DNA viral, por no cumplir criterios de trasplante ni estar indicado el tratamiento antiviral. El número de pacientes que tenían serología positiva al VHC fue 18 (10,9\%), la cuantificación de la carga viral mediante PCR fue efectuada en 7 pacientes, de los cuales seis $(85,7 \%)$ presentaron una carga viral superior a 200.000 copias $/ \mathrm{ml}$, no efectuamos la medición de la carga viral en el resto de los pacientes porque no tenían criterios de tratamiento ni de posible trasplante hepático. La presencia de coinfección VHD más VHB existió en dos casos, siendo en ambos casos el DNA viral del VHB positivo $(1,2 \%)$. La coinfección VHB más VHC existió también en dos casos, siendo el DNA viral del VHB como el RNA viral del VHC positivos $(1,2 \%)$. A la totalidad del grupo estudiado se les realizó determinaciones analíticas que nos permitieron descartar otras posibles etiologías de su hepatopatía. El porcentaje de pacientes que presentaron uno o más episodios de complicación de su cirrosis fue del 39\% lo que representa a 65 individuos (Fig. 1). Mientras, las complicaciones principales no relacionadas fueron 12 episodios de pancreatitis aguda, 
23 episodios de hemorragia digestiva alta no varicosa y 7 episodios de delirium tremens. Al calcular el índice de Maddrey, apreciamos al comienzo del estudio unos valores de 6,5 $\pm 7,8$ y

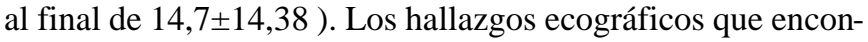
tramos tanto al comienzo del estudio como al final del mismo se aprecian en la tabla I. Destacando fundamentalmente el aumento de cirrosis y la aparición de lesiones ocupantes de espacio que, tras estudio histológico, fueron diagnosticadas de hepatocarcinomas $(8,5 \%)$. Detectamos que el inicio del hábito enólico fue más precoz en los hombres que en las mujeres, con significación estadística $(17,6(4,4)$ años vs 31,2 $(20,4)$ años) ( $\mathrm{p}<0,0001)$, también observamos que: el consumo diario de alcohol fue mayor en hombres que en mujeres $(169,4$ $(117,9)$ gramos diarios vs $72(49,3)$ gramos diarios) $(\mathrm{p}<0,05)$, que los hombres presentaron una mayor duración de su enolismo respecto a las mujeres $(17(12,2)$ años vs $37(12,5)$ años) ( $\mathrm{p}=0,003)$, y que el consumo acumulado de alcohol a lo largo de la vida también fue mayor en los hombres que en las mujeres $(2034061,4(1629781,7)$ gramos vs $292000(313697,3)$ gramos) $(\mathrm{p}<0,0001)$ estando este correlacionado con el TP al final del estudio $(r=+0,391)$. Encontramos que el consumo diario de gramos de alcohol estuvo correlacionado con: el aspecto ecográfico del hígado $(\mathrm{r}=+0,283)$ y con el índice de

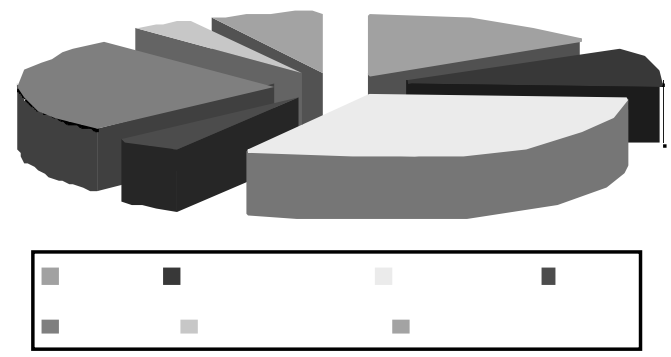

Fig. 1. Complicaciones de la cirrosis.

\section{TABLA I}

\begin{tabular}{|c|c|c|c|c|}
\hline \multicolumn{5}{|c|}{ TABLA I } \\
\hline \multicolumn{5}{|c|}{ EVOLUCIÓN SIGNOS ECO GRÁFICOS } \\
\hline Hallazgos ecográficos & Inicio & & Final & \\
\hline H. crónica & 63 & $38,4 \%$ & 64 & $39 \%$ \\
\hline Cirrosis & 48 & $29,2 \%$ & 70 & $42,7 \%$ \\
\hline Esteatosis & 40 & $24,4 \%$ & 23 & $14 \%$ \\
\hline Normal & 13 & $7,9 \%$ & 7 & $4,2 \%$ \\
\hline Litiasis B. & 44 & $26,8 \%$ & 58 & $35,3 \%$ \\
\hline Esplenomegalia & 83 & $50,6 \%$ & 85 & $51,8 \%$ \\
\hline Vel. V porta disminuida & 82 & $50 \%$ & 94 & $57,3 \%$ \\
\hline V. porta dilatada & 93 & $56,6 \%$ & 95 & $57,9 \%$ \\
\hline Hepatocarcinoma & 3 & $1,8 \%$ & 12 & $8,5 \%$ \\
\hline
\end{tabular}

Maddrey al final del estudio $(\mathrm{r}=+0,407)$. Hemos podido observar que de forma estadísticamente significativa $(\mathrm{p}<0,05)$, aquellos pacientes que tenían un mayor consumo acumulado de alcohol a lo largo de la vida y mayor cantidad de gramos de alcohol /día ingeridos, muestran con mayor incidencia al final del estudio: patrón de hepatitis crónica, signos ecográficos de cirrosis, disminución de la velocidad del flujo de la vena porta y aparición de algún tipo de descompensación de su hepatopatía. El Tiempo de Protrombina al inicio del estudio estuvo correlacionado con el aspecto ecográfico del hígado $(r=+0,301)$.

\section{DISCUSIÓN}

El consumo de alcohol de nuestros pacientes fue superior al de un grupo de pacientes franceses alcohólicos, en el que el consumo diario de alcohol era de 118 (81) gramos, de la misma manera el número de años de enolismo fue inferior en dicha población, concretamente 22 (13) años (8). Del total de pacientes, 164, abandonaron el hábito enólico 72 después de la primera consulta, constatado mediante encuesta y/o determinaciones analíticas, lo cual está en consonancia con los resultados de un estudio de la evolución del alcoholismo en Moscú, tras una campaña en pro de la abstinencia (9). El porcentaje de pacientes con Hepatocarcinoma fue superior ligeramente al 2,6\% comunicado por un grupo procedente de Sydney, Australia (10). En nuestro estudio, la mayoría de los pacientes pertenecían a un nivel socioeconómico bajo, esta observación está acorde con los resultados de un estudio sueco (11), aunque nuestro hospital pertenece al sistema público , y siempre hay un importante número de pacientes que acude al sistema privado. La prevalencia de anticuerpos positivos frente al virus $\mathrm{C}$ de la Hepatitis y de la positividad del AgHBs, fue ligeramente superior a la comunicada por un grupo israelí, que presentaba una seropositividad frente al virus $\mathrm{C}$ de la hepatitis del 7,6\% y una prevalencia de la presencia de AgHBs del 4,8\% (12). Sin embargo, un estudio sudamericano sitúa la cifra de AcVHC + en un $20 \%$ y la cifra de anticuerpo anti-core de la hepatitis B en un 13,9\% (13). El porcentaje de pacientes que sufrieron un episodio de Delirium Tremens $(4,2 \%)$ fue menor que el porcentaje aportado por un grupo procedente de Lübeck en Alemania, dónde alcanzaba un $12,8 \%$ (14). Hemos apreciado que el porcentaje de pacientes con patología pancreática era del 7,3\% inferior al 19\% publicado en otros estudios (15).

Encontramos un mayor porcentaje de hombres $(91,5 \%)$ consumidores de alcohol, debido probablemente a que la mujer no acude al médico por temor a que se le reconozca socialmente su hábito enólico, siendo los años de enolismo medio de 35,4 $(13,5)$ años. Ninguna mujer consumió menos de 20 gramos diarios de alcohol. Se aprecia un ligero predominio de pacientes que pertenecen al medio rural $(56,7 \%)$ y poseían un nivel cultural bajo (73\%) no hallándose ningún paciente con un nivel alto lo que nos hace pensar el poco uso de la sanidad pública por este colectivo social. La edad media de inicio del hábito enólico fue 18,6 $(7,36)$ años, y el porcentaje de pacientes que presentaron uno o más episodios de complicación de su cirrosis fue del 39,\% predominando la ascitis con un 33,5\%. El inicio del hábito enólico fue más pre$\mathrm{coz}$ en los hombres que en las mujeres de forma estadísticamente significativa $(\mathrm{p}<0,0001)$. Del mismo modo el consumo 
diario de alcohol, la duración de su enolismo y el consumo acumulado de alcohol a lo largo de la vida fue mayor en los hombres que en las mujeres. Este predominio del sexo masculino en todos los parámetros anteriormente citados, habría que contrastarlos en una muestra donde hubiese mayor número de

\section{Bibliografía}

1. Coates RA, Halliday ML, Rankin JG, Feinamn SV, Fisher MM. Risk of fatty infiltration or cirrhosis of the liver in relation to ethanol consumption: a case-control study. Clin Invest Med 1986; 9: 26-32.

2. Parés A. Epidemiología del consumo de alcohol y de la enfermedad hepática alcohólica. En: Rodes J, Chantar C, Editores. Actualidades en gastroenterología y hepatología. Barcelona: Prous Editores, 1995.

3. Edmondson HA. Pathology of alcoholism Am J Clin Pathol 1980; 74(5): 725-42.

4. Becker PU, Deis A, Sorensen TI, Gronbaek MN, Borch-Johnsen K, Muller CF, et al. Alcohol intake and risk of liver disease--significance of gender. A population study. Ugeskr Laeger 1999; 159(24): 3782-6.

5. Parés A, Tresserras R, Núñez I, Cerralbo M, Plana P, Pujol FJ, et al. Prevalencia y factores asociados a la presencia de esteatosis hepática en varones adultos aparentemente sanos.Med Clin(Barc) 2000; 114: 561-5.

6. Watson RR, Mohs ME, Eskelson C, Sampliner RE, Hartmann B. Identification of alcohol abuse and alcoholism with biological parameters. Alcohol Clin Exp Res 1986; 10(4): 364-85.

7. Corrao G, Bagnardi V, Zambon A, Arico S. Exploring the dose-response relationship between alcohol consumption and the risk of several alcohol-related conditions: a meta-analysis. Addiction 1999; 94(10): 1551-73.

8. Naveau S, Borotto E, Giraud V, Bardou M, Aubert A, Lazizi Y, et al Descriptive epidemiology of patients with alcoholic liver disease hospitalized in a hepato-gastroenterology service . Gastroenterol Clin Biol 1999; 23(5): 544-51. mujeres, si bien en nuestro estudio no ha existido manipulación alguna a la hora de incluir a los pacientes. Del total de la muestra un 7,3\% (12 pacientes) presentaban patología psiquiátrica, y a 14 pacientes $(8,5 \%)$ se incluyeron en protocolo de trasplante hepático.

9. Nemtsov AV. Alcohol-related harm and alcohol consumption in Moscow before, during and after a major anti-alcohol campaign. Addiction 1998; 93(10): 1501-10.

10. Larcos G, Sorokopud H, Berry G, Farrell GC. Sonographic screening for hepatocellular carcinoma in patients with chronic hepatitis or cirrhosis: an evaluation. AJR Am J Roentgenol 1998; 171(2): 433-5.

11. Hemmingsson T, Lundberg I, Romelsjo A, Alfredsson L. Alcoholism in social classes and occupations in Sweden. Int J Epidemiol 1997; 26(3): 584-91.

12. Srugo I, Shinar E, Bar-Shany S, Amos L. Hepatitis B and C markers among alcoholics in Israel: high incidence of HCV infection. Eur J Epidemiol 1998; 14(4): 333-7.

13. Curciarello J, Apraiz M, Chiera A, Castelletto R, Vassia MA, Barbero $\mathrm{R}$, et al. Hepatitis $\mathrm{B}$ and $\mathrm{C}$ virus in chronic alcoholic patients: prevalence and influence on liver injury. Acta Gastroenterol Latinoam 1996; 26(4): 211-4.

14. Gerke P, Hapke U, Rumpf HJ, John U. Alcohol-related diseases in general hospital patients. Alcohol Alcohol 1997; 32(2): 179-84.

15. Hastier P, Buckley MJ, Francois E, Peten EP, Dumas R, Caroli-Bosc $\mathrm{FX}$, et al. A prospective study of pancreatic disease in patients with alcoholic cirrhosis: comparative diagnostic value of ERCP and EUS and long-term significance of isolated parenchymal abnormalities. Gastrointest Endosc 1999; 49(6): 705-9. 